Bull. Mater. Sci., Vol. 14, No. 1, February 1991, pp. 1-10. (C) Printed in India.

\title{
Effect of indium concentration on the internal oxidation of a silver-tin alloy
}

\author{
A VERMA and T R ANANTHARAMAN \\ Thapar Corporate Research and Development Centre, Patiala 147001 , India \\ MS received 26 May 1990; revised 14 January 1991
}

\begin{abstract}
The investigation reported here was undertaken to study the beneficial influence of indium on the internal oxidation behavieur of silver-tin alloys in the context of electrical contact material development. Five compositions of varying indium content, namely Ag-6Sn-xIn $(x=2 \cdot 0,2 \cdot 5,3 \cdot 0,3 \cdot 5,4 \cdot 0 \mathrm{wt} \%)$ were prepared and internally oxidized at different temperatures and oxygen pressure. The kinetics of internal oxidation was studied and an attempt made to correlate the same with microstructure.
\end{abstract}

Keywords. Electrical contact materials; internal oxidation; silver-tin oxide-indium oxide.

\section{Introduction}

It has recently been established that silver-cadmium oxide ( $\mathrm{Ag}-\mathrm{CdO})$ composites are suitable for switching devices operating in the range of 50-5000 amp (Schroder 1987). However, because of the toxic nature of cadmium and the increasingly stricter environmental regulations, silver-tin oxide $\left(\mathrm{Ag}-\mathrm{SnO}_{2}\right)$ has recently emerged as a possible substitute for $\mathrm{Ag}-\mathrm{CdO} . \mathrm{Ag}-\mathrm{SnO}_{2}$ has superior erosion resistance and anti-welding characteristics (Sabairi et al 1982).

Two manufacturing techniques have generally been adopted for $\mathrm{Ag}-\mathrm{SnO}_{2}$ materials, namely, powder metallurgy (PM) (Yamasaki et al 1980) and internal oxidation (IO) (Bohm et al 1980). However, the latter process suffers from the problem of high inter-diffusion coefficient of tin in silver. It has been established that a high tin content $(>4 \mathrm{wt} \%)$ in silver is not amenable to internal oxidation as it forms an impervious layer of tin oxide inhibiting further diffusion of oxygen in the alloy (Shen and Gould 1987). In order to facilitate internal oxidation, several additives such as bismuth, copper and indium have been tried with different measures of success. Among these only the indium-bearing silver-tin alloy has been commercially exploited to its full potential. Indium prevents the formation of dense oxide bands of $\mathrm{SnO}_{2}$ and enables oxygen to diffuse at a faster rate into the silver-tin alloy.

The kinetics of the internal oxidation process is given by Wagner's (1959) parabolic relationship viz

$$
x^{2}=k \cdot t
$$

where $x$ is the thickness of the internally oxidized zone after time $t$ and $k$ the scaling constant given by

$$
k=2 \cdot\left(D_{0} / v\right)\left(N_{0} / N_{m}\right)
$$

where $D_{0}$ is the diffusion coefficient of oxygen in the alloy; $v$ the oxygen-to-metal atom ratio in oxide, $N_{0}$ the oxygen concentration on the surface of alloy and $N_{m}$ the concentration of the metal to be oxidized. 
Equation (2) is based on the solution of the diffusion equation with appropriate boundary conditions and suitable approximations. However, the measured thickness of the oxidized zone and theoretically calculated values differ if the reactive solute also provides significant diffusion. Under these conditions the expression remains parabolic, but the scaling constant also includes the diffusion coefficient of reactive metals. For a given time, the depth of internal oxidation is less than that calculated from (1), and the product enriches the solute in the reacted zone.

Due to enrichment, dense oxide particles in increasingly greater concentration are formed, which reduce the total cross-sectional area available for oxygen diffusion, leading to reduced depth of internal oxidation. However, while dealing with the internal oxidation of ternary alloy systems such as silver-tin-indium, one also has to consider the relative stability of the oxides of solutes. Indium forms a much stabler oxide than tin (free energy of formation of $\operatorname{In}_{2} \mathrm{O}_{3}$ at $298 \mathrm{~K}$ is $198.55 \mathrm{kcal} / \mathrm{mol}$ and that of $\mathrm{SnO}_{2}-124.2 \mathrm{kcal} / \mathrm{mol}$ ). Therefore, the uniformly distributed elemental indium will be oxidized at a lower oxygen concentration than would tin. Once the $\mathrm{In}_{2} \mathrm{O}_{3}$ particles are formed, they become nuclei for the precipitation of tin oxide and may also enhance the rate of precipitation. The insoluble sub-microscopic particles greatly increase the amount of high energy interface regions. It is well established that the diffusion rate along grain boundaries and high energy interface is much higher than within the grain. Thus, the actual depth of internal oxidation will be decided by many competing processes as described above.

It is apparent from the foregoing equations that oxygen pressure, temperature and concentration of active metal influence the scaling constant and hence the overall kinetics of the process. The scaling constant is expressed in terms of activation energy $(Q)$ of the internal oxidation process (GroBe et al 1966) through an Arrhenius type of relation viz.

$$
k=A \exp -(Q / R T) \text {. }
$$

The activation energy of the process is a fixed quantity for a particular composition and oxygen pressure.

The selection of composition in this work was based on the contact property data reported by different authors and optimum properties found by them in different compositions (Shen and Gould 1987; Honda 1979). It has been established that the maximum indium percentage should be in the range of $2-4 \mathrm{wt} \%$ for optimum contact properties. More than $4 \mathrm{wt} \%$ indium affects the sticking resistance adversely, whereas $<2 \mathrm{wt} \%$ indium is not very effective in facilitating the process of internal oxidation of tin. The optimum tin content for satisfactory performance and processing has been established to be in the range of 4-10 wt \% (Honda 1979).

\section{Experimental}

Five alloys with compositions $(100-\overline{6+x})$ Ag-6Sn- $x \operatorname{In}(x=2 \cdot 0,2 \cdot 5,3 \cdot 0,3 \cdot 5,4 \cdot 0)$ were melted under vacuum in an induction furnace. The as-cast alloys were rolled in the form of sheets of $4 \mathrm{~mm}$ thickness. These sheets were homogenized at $650^{\circ} \mathrm{C}$ for $15 \mathrm{~h}$. Subsequently, cylindrical pellets of $7.6 \mathrm{~mm}$ diameter were punched out of the sheet. Each pellet was cleaned by polishing on 240 grit emery paper to remove 
the scale formed during punching and handling. X-ray diffraction of the homogenized alloy was taken to determine the combined terminal solubility of indium and tin in silver.

The above pellets were internally oxidized at three different tempcratures, i.e. 823, 923 and $1023 \mathrm{~K}$ at atmospheric oxygen pressure $\left(0.21 \mathrm{~kg} / \mathrm{cm}^{2}\right)$ and elevated oxygen pressure $\left(6.0 \mathrm{~kg} / \mathrm{cm}^{2}\right)$. In order to carry out internal oxidation at elevated oxygen pressure a heat-resistant steel tube with one end closed was used. The test pieces were internally oxidized for different lengths of time, namely 4, 9, 16 and 25 hours.

The specimens were cut by a diamond wafering blade and polished to observe the longitudinal section of the pellets. Thickness of the oxidized zone was measured to the accuracy of $0.01 \mathrm{~mm}$ with the help of a micrometer attached to a microhardness tester. Electrical conductivity and hardness values were measured and the microstructure examined at the depth of $0.05 \mathrm{~mm}$ from surface, along the direction of oxygen diffusion.

\section{Results and discussion}

Figure 1 showing the variation of lattice constant of silver-tin-indium alloys with varying percentage of indium suggests that out of the five chosen alloys only three, namely, Ag-6Sn- $x$ In $(x=2 \cdot 0,2 \cdot 5,3 \cdot 0)$ were single phase alloys. Ag-6Sn alloy without any additive showed the formation of a dense oxide band following internal oxidation (figure 2) at $923 \mathrm{~K}$ and $0.21 \mathrm{~kg} / \mathrm{cm}^{2}$ oxygen pressure. The formation of bands is attributed to the high diffusion coefficient of tin in silver. However, addition of indium circumvents this problem, and it was possible to internally oxidize tin even at $823 \mathrm{~K}$, though at a sluggish rate. Indium checks the diffusion of tin since the free energy of formation of indium oxide is more negative than tin oxide. Thus indium oxide is formed first and acts as a nucleation site for formation of the tin oxide.

Variation of oxidation depth with time at different temperatures for two different oxygen pressures, namely, $0.21 \mathrm{~kg} / \mathrm{cm}^{2}$ and $6.0 \mathrm{~kg} / \mathrm{cm}^{2}$ is plotted in figures $3(\mathrm{a})$ and 3 (b) respectively. It may be observed that the parabolic law of internal oxidation is obeyed at all temperatures as well as atmospheric and elevated oxygen pressures. The observed high rate of internal oxidation at higher temperatures is in conformity with the theory proposed for internal oxidation. However, the rate of internal

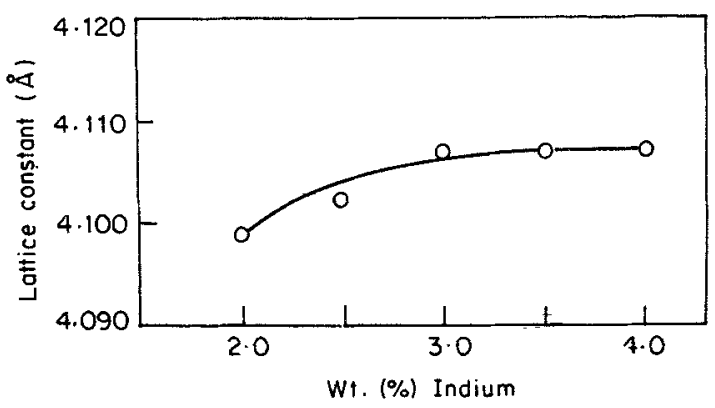

Figure 1. Variation of lattice constant of silver-tin alloy with varying concentration of indium. 


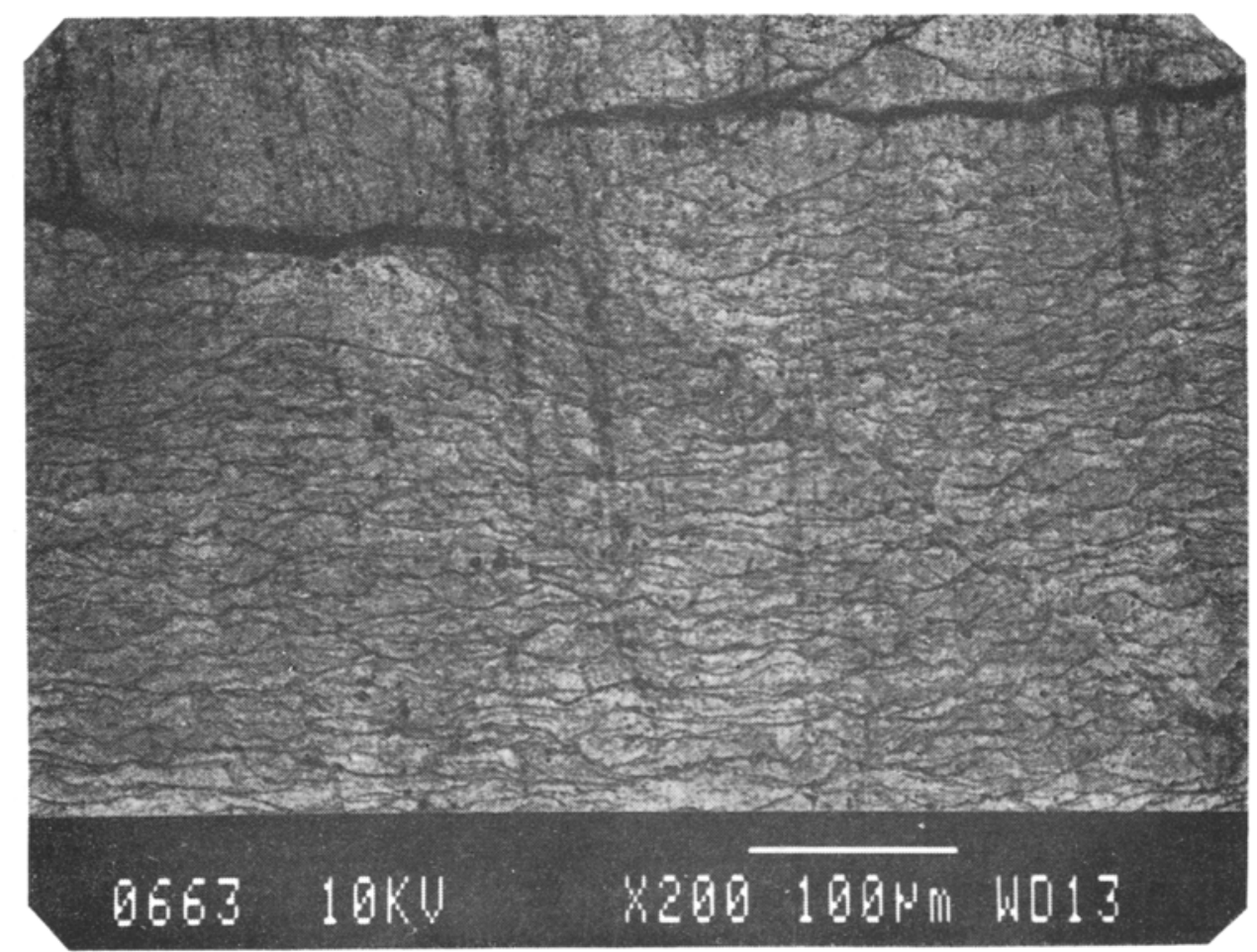

Figure 2. Oxide hands in internally oxidized Ag-6.0 Sn alloy.

oxidation at $823 \mathrm{~K}$ drops down substantially at lower oxygen pressure. It may also be noted that at a given temperature the rate of oxidation at $6.0 \mathrm{~kg} \mathrm{~cm}^{2}$ is an order of magnitude faster than that of air oxidation. This can be attributed to higher solubility of oxygen at higher pressure (Sievert's law) in silver which provides a steeper concentration gradient of oxygen from the surface to the bulk and favourably affects the internal oxidation kinetics.

Rate constants at different temperatures and pressures were determined for all compositions (figures $4 a$ and 4 b). It may be observed that the rate constant decreases almost linearly in the range $8-10 \mathrm{wt} \%$ of the total alloy content. This decrease is more marked at the higher oxidation temperatures. A higher fall in rate constant with composition at higher temperatures may be explained on the basis of faster diffusion of alloying elements towards the advancing oxidation front and formation of dense oxides which retard subsequent oxidation. It may be noted that the fall in rate constant with composition at higher temperature is more steep for the lower oxidation pressure $\left.10.21 \mathrm{~kg} \mathrm{~cm}^{2}\right)$. This corroborates the assumption that faster diffusion of tin and indium is responsible for greater variation of rate constant with composition at higher temperature.

Based on the observed variation in rate constant with composition and temperature the rate constant values were plotted against the inverse of temperature (figures $5 \mathrm{a}$ and $5 \mathrm{~b}$ ) to evaluate the activation energy for the internal oxidation process. The variation of activation energy with composition for $0.21 \mathrm{~kg} / \mathrm{cm}^{2}$ and $60 \mathrm{~kg} / \mathrm{cm}^{2}$ oxygen pressures is plotted in figures $6 \mathrm{a}$ and $6 \mathrm{~b}$ respectively. It is seen 

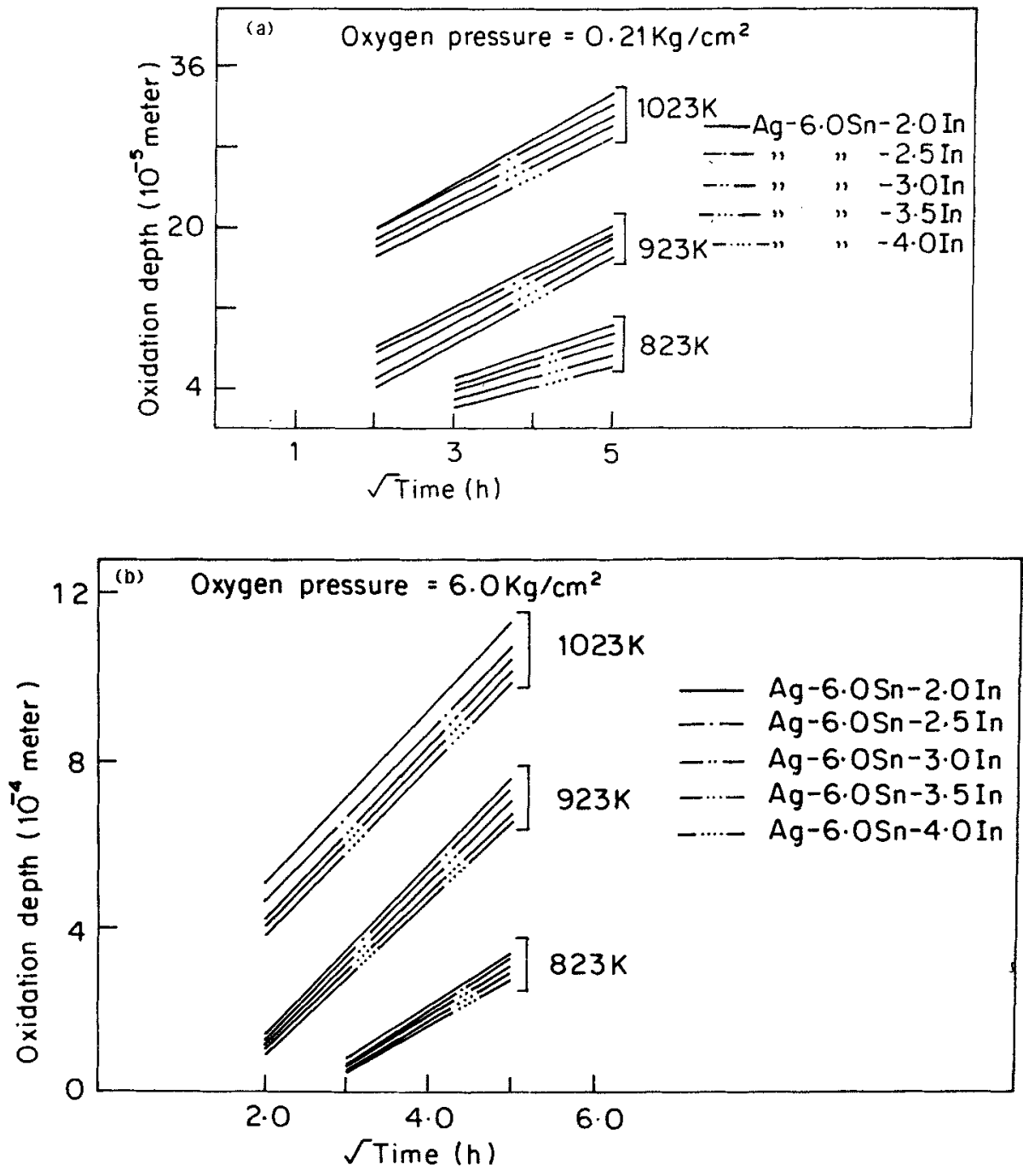

Figure 3. Variation in depth of oxidation with temperature, time and composition. a. oxygen pressure $=0.21 \mathrm{~kg} / \mathrm{cm}^{2}$, b. oxygen pressure $=6.0 \mathrm{~kg} / \mathrm{cm}^{2}$.

that the activation energy of the process increases with alloy concentration. This increase may be ascribed to the reduction in the fractional volume of silver available for oxygen to diffuse as the volume percentage of alloying elements increases. Further, oxides being very dense, the rate of diffusion of oxygen through oxides is comparatively negligible.

Scanning electron micrographs (SEM) were taken along the direction of oxygen diffusion for all compositions. Micrographs $7 \mathrm{a}$ and $7 \mathrm{~b}$ show the size and shape of the oxide dispersion at $0.21 \mathrm{~kg} / \mathrm{cm}^{2}$ and $6.0 \mathrm{~kg} / \mathrm{cm}^{2}$ respectively. These micrographs have been taken at equal distances from the surface in view of the gradual coarsening of oxides from the surface to the bulk. It may be noted that coarsening of oxide particles occurs as the indium percentage is varied over the range of 2- 

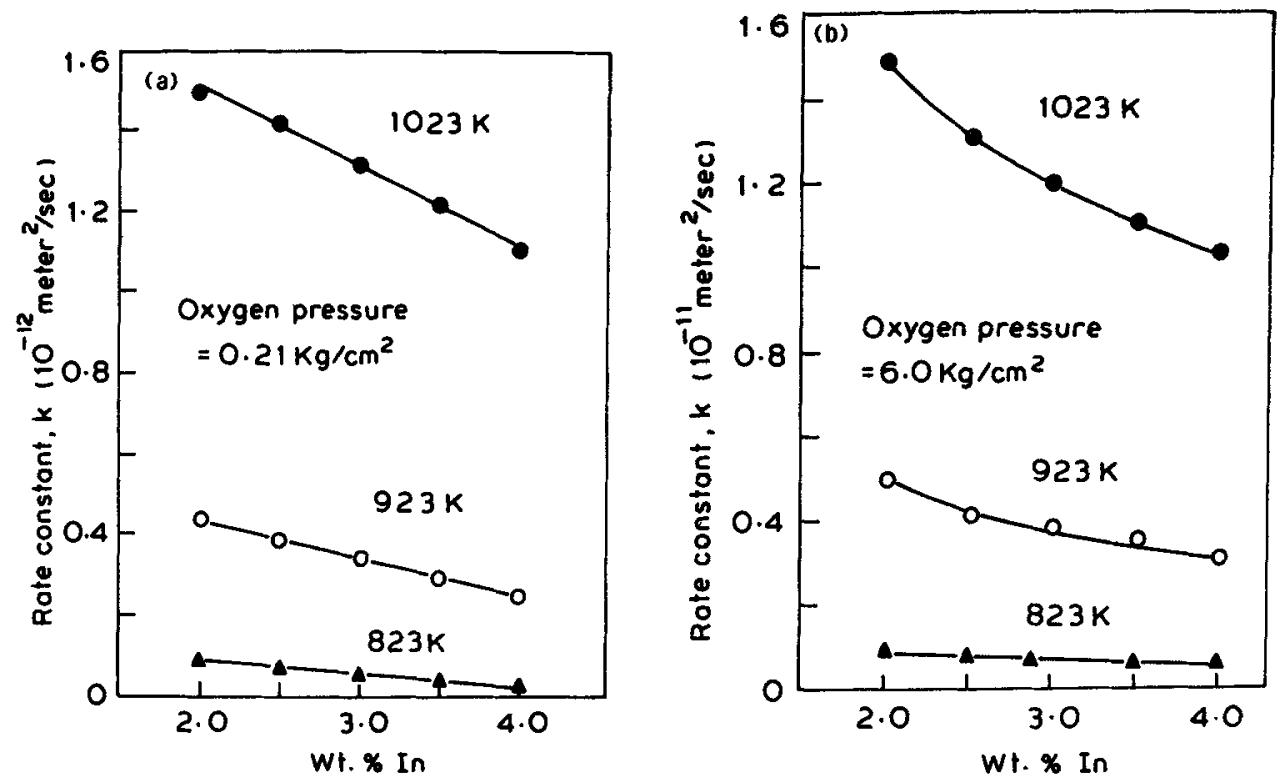

Figure 4. Variation in rate constant with composition and temperature. a. oxygen pressure $=0.21 \mathrm{~kg} / \mathrm{cm}^{2}$, b. oxygen pressure $=6.0 \mathrm{~kg} / \mathrm{cm}^{2}$.
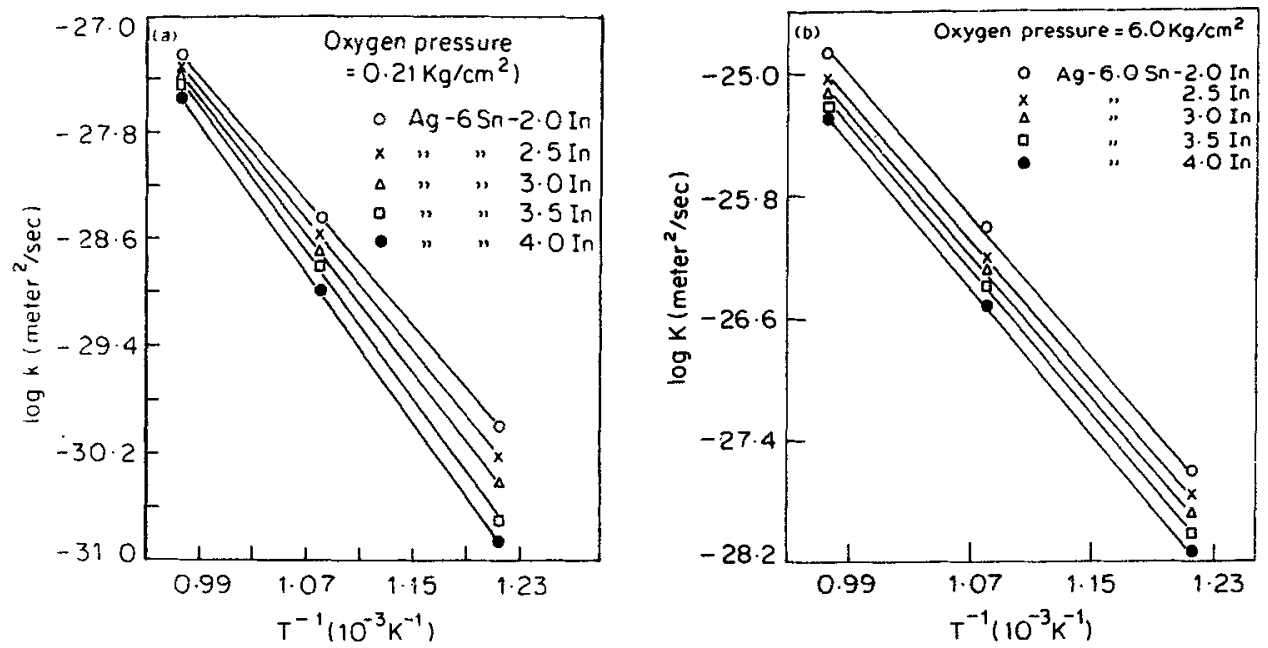

Figure 5. $\log K$ vs $(1 / T)$ plot for Ag-6Sn-xIn $(x=2 \cdot 0,2 \cdot 5,3 \cdot 0,3 \cdot 5,4 \cdot 0)$ alloy. a. oxygen pressure $0.21 \mathrm{~kg} / \mathrm{cm}^{2}$, b. oxygen pressure $6.0 \mathrm{~kg} / \mathrm{cm}^{2}$.

$4 \mathrm{wt} \%$. It is also apparent from the micrographs that the fractional volume of silver is substantially reduced over the compositional range. It is quite likely that oxides are enriched with increase in indium concentration. However, this could not be confirmed in the absence of an energy-dispersive spectrometer. A thin $(1-2 \mu \mathrm{m})$ oxide-free silver layer following internal oxidation is observed in all the samples. 

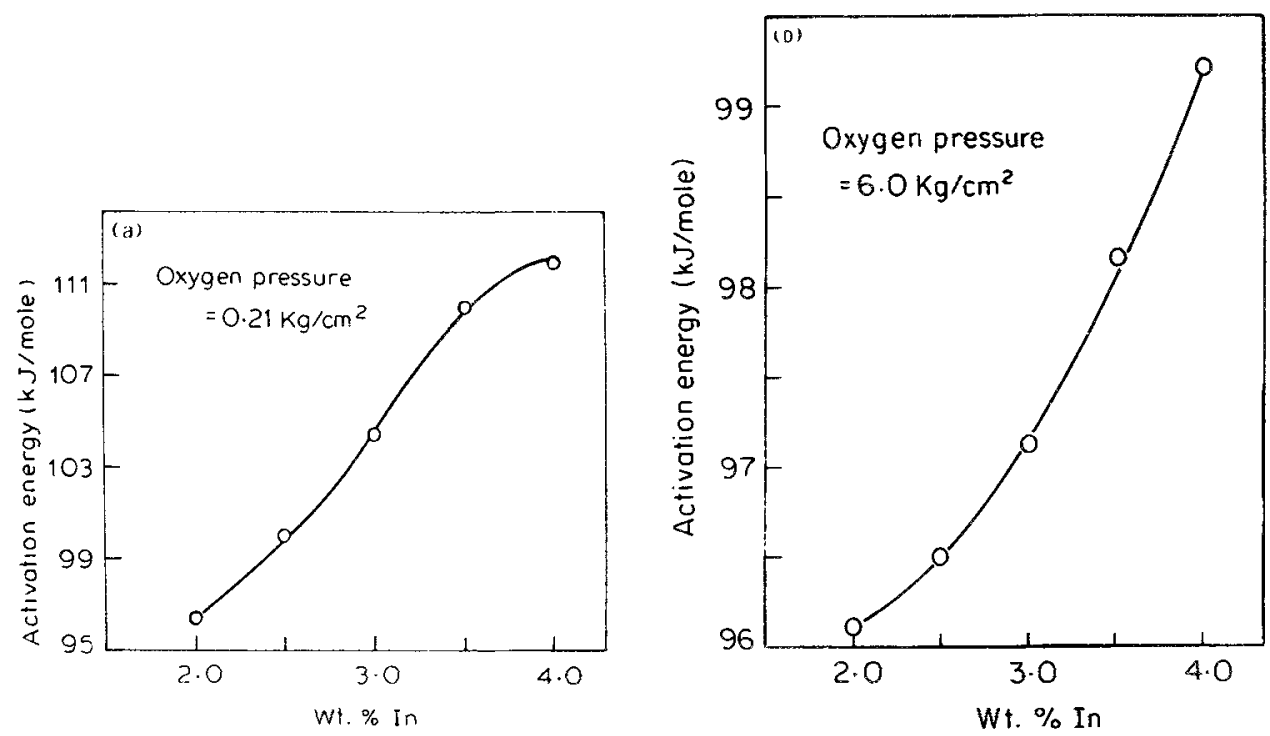

Figure 6. Variation of activation energy of internal oxidation process for Ag-6Sn-x In $(x=2 \cdot 0,2 \cdot 5,3 \cdot 0,3 \cdot 5,4.0)$ alloys. a. oxygen pressure $0.21 \mathrm{~kg} / \mathrm{cm}^{2}$, b. oxygen pressure $6.0 \mathrm{~kg} / \mathrm{cm}^{2}$.

The mechanism of formation of these layers has been attributed to the onset of diffusion creep owing to the formation of bulky oxides (Machert 1986). This observation gives support to the idea of enrichment of oxides as the indium content is increased.

The difference in size of oxide particles at different oxygen pressures is evident in the scanning electron micrographs. At lower oxygen pressure $\left(0.21 \mathrm{~kg} / \mathrm{cm}^{2}\right)$ the size of oxide particles is coarser than at higher oxygen pressure. This can be attributed to the modified nucleation and growth kinetics of oxide particles at higher oxygen pressure. Due to a steeper oxygen concentration gradient, the oxidation front moves at a faster rate. As the oxide front sweeps through the alloy, it nucleates oxide particles and before these nucleated oxide particles have an opportunity to grow by the counter diffusion of oxide elements, fresh nucleation takes place ahead of the previous nucleation site. However, at lower oxidation pressure, the rate of advancing oxidation front is reduced and consequently the growth of oxide particles is appreciable. Further, since the heat of formation of tin oxide $\left(\mathrm{SnO}_{2}\right)$ and indium oxide $\left(\mathrm{In}_{2} \mathrm{O}_{3}\right)$ at $298 \mathrm{~K}$ is -124.2 and $-198.55 \mathrm{kcal} / \mathrm{mole}$, respectively, it is quite likely that indium oxide acts as nucleating agent for the growth of the tin oxide.

Table 1 gives the physical properties relevant for the characterization of electrical contact materials. The trend in change of electrical conductivity with volume fraction of oxides is in conformity with the law of mixtures. However, the theoretical values do not match with experimental results. This discrepancy may arise due to two reasons. Firstly, if the enrichment of oxide does take place, the volume fraction of silver is correspondingly reduced. Secondly, the precipitation of oxides changes the scattering characteristics of silver. Both these effects impair the conductivity values. It may be noted that the hardness value does not increase in step with the increase in volume fraction of oxides. This behaviour may be 


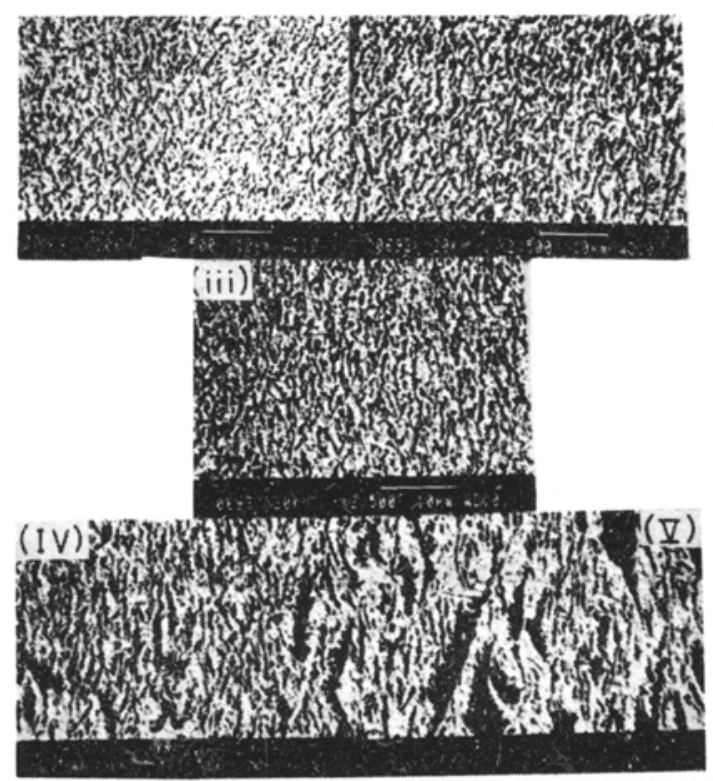

(a)

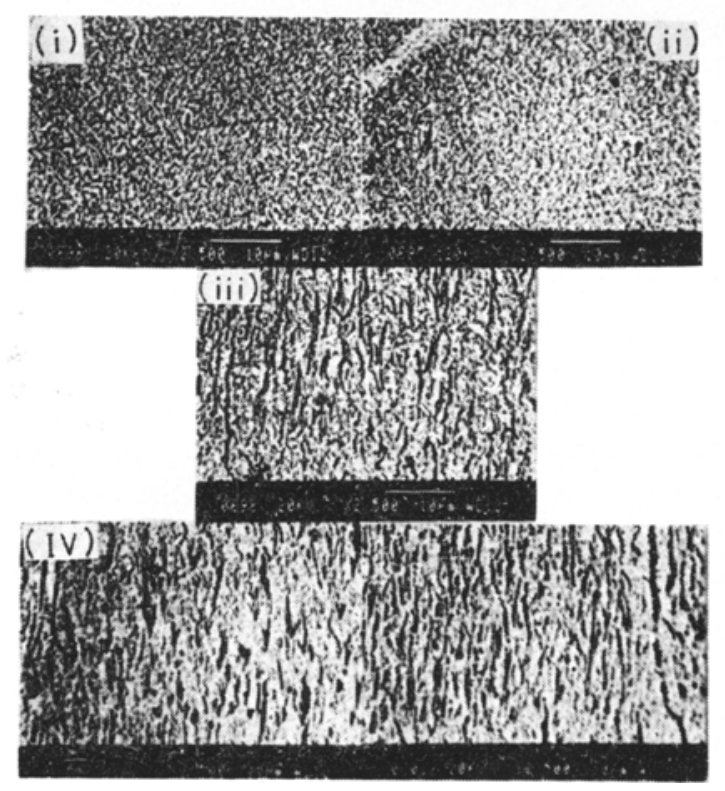

(b)

Figure 7. Scanning electron micrographs taken along the moving oxygen front for Ag$6 \operatorname{Sn}-x \operatorname{In}(x=2 \cdot 0,2 \cdot 5,3 \cdot 0,3 \cdot 5,4 \cdot 0)$ alloys. a. oxygen partial pressure $0 \cdot 21 \mathrm{~kg} / \mathrm{cm}^{2}$. b. oxygen partial pressure $6.0 \mathrm{~kg} / \mathrm{cm}^{2}$.

accounted for the coarsening of oxide particles with increased volume fraction (see micrographs $7 \mathrm{a}$ and $7 \mathrm{~b}$ ). Coarsening of oxides leads to increase in interparticle spacing and a consequent decrease in barriers arresting the motion of dislocations. The difference in hardness values between samples oxidized at different oxygen 


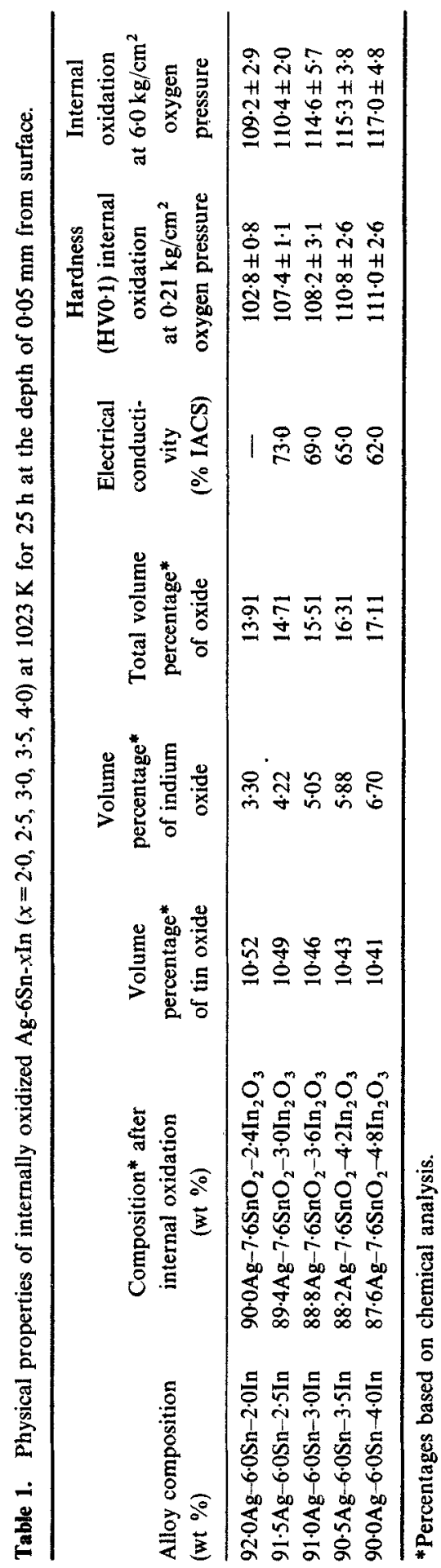


pressures may also be explained by the easier movement of dislocations in coarser microstructures.

\section{Conclusion}

The following points emerge from the present study of the role of indium in the internal oxidation of a silver-tin alloy under different process conditions:

(i) Ag-6Sn- $x \operatorname{In}(x=2.0,2.3,3.0 \mathrm{wt} \%)$ alloys are single phase and amenable to the internal oxidation process. Higher percentage of indium does not facilitate the internal oxidation process. For indium concentrations exceeding $3 \mathrm{wt} \%$, coarsening of oxide particles occurs, which may not be favourable from the point of view of contact properties.

(ii) Higher oxygen pressure remarkably increases the rate of internal oxidation and leads to refinement of the dispersed oxide. The former is relevant from the view point of application, while the latter is likely to impart better anti-welding and antierosion properties to the product.

(iii) Although internal oxidation at higher partial pressure of oxygen leads to refinement of dispersoids, the hardness values do not increase appreciably. This is desirable in the context of making the required shapes.

\section{References}

Bohm W, Lindmayer M and Classing M 1980 Proc. 27th Annual Meeting of the Holm Conference on Electrical Contacts 51

GroBe J, Moser T and Rothkegel B 1986 Proc. 13th Int. Conf. on Electric Contacts (Switzerland: Lausanne) 211

Honda T 1979 Alloy materials for electrical contact breakers, Japanese Patent No. 54-100922

Machert J R 1986 Met. Trans. A 17a 746

Sabairi K, Tsuji H and Tsuchiya K 1982 Proc. 28th Annual Meeting of the Holn Conference on Electrical Contacts 77

Schroder K-H 1987 IEEE Trans. CHMT-10 127

Shen Y-S and Gould L J 1987 IEEE Trans. CHMT-4 157

Wagner C 1959 Electrochemistry 63772

Yamasaki H, Oda S and Miyakawa K 1980 Proc. 10th Int. Conf. on Electrical Contact Phenomena 799 